\title{
ON CERTAIN DYNAMICAL SYSTEMS WITH POINTS OF PEANO
}

\author{
BY MARIE CHARPENTIER*
}

1. Introduction. Recently I have studied differential systems of the form

$$
\frac{d x_{i}}{d t}=X_{i}\left(x_{j}\right), \quad(i, j=1, \cdots, n)
$$

in which the point $\left(x_{i}\right)$ is restricted to lie in a rectangular domain of $x_{i}$ space while the $X_{i}$ are merely taken to be continuous. $\dagger$ As is well known, there may exist in this case more than one integral curve through certain "points of Peano."

Such differential systems have an obvious physical interest, for they represent the simplest possible extension of the ordinary dynamical type, in which a certain indeterminacy of motion may exist; in the dynamical case the manifold $M$ is closed instead of rectangular. A first fundamental question in this direction is as to whether there exists an analog of the central motions as defined by Birkhoff. $\ddagger$

In the present paper, I have commenced with the case of most interest, in which there is uniqueness for decreasing $t . \S \mathrm{An}$ analogous set of central motions is defined in this case and these are determined for the sphere $(n=2)$.

2. Central Motions with a Condition of Uniqueness for Decreasing $t$. (a). Consider a point $P$ at an instant $t_{0}$ on the manifold $M$. Let us define for any $\epsilon>0$, an open molecule $\sigma$ of diam-

* International Research Fellow.

$\dagger$ Bulletin des Sciences Mathématiques, vol. 54 (1930), p. 203. Comptes Rendus, vol. 191 (1930), p. 912; and vol. 192 (1931), p. 913. Bulletin International de l'Académie Polonaise, 1931, p. 191, and Mathematica (Cluj), vol. 5, pp. 65-99.

\$ See, in particular, Dynamical Systems, Colloquium Publications of this Society, vol. 9, p. 189; and G. D. Birkhoff and P. A. Smith, Journal de Mathématiques, 1928, p. 345 .

$\S$ That is, if we consider the curves of motion passing through $P$ for $t=t_{0}$, they coincide for $t<t_{0}$. For the study of this case in the plane see p. 87 of my Mathematica paper. 
eter $\epsilon$, containing $P$. Each point of the molecule is supposed to move in all admissible directions as time increases, but, of course, these points will always fill a continuum.

In what follows we shall use the following fundamental property of the motions. Let $(P)_{t}$ be the set of points to any of which $P$ may have moved after a time $t,(P)_{t}$ being by hypothesis a single point for $t<0$; for any point $P$, any $t_{0}>0$, and any $\epsilon>0$, there is a $\delta>0$ such that, if $Q$ is within a distance $\delta$ of $P$, then any point of $(Q)_{t}$ is within $\epsilon$ of some point of $(P)_{t}$ for $|t| \leqq t_{0}$. It follows that any set $(P)_{t}$ is closed.

(b). Consider now the above defined molecule $\sigma$. If $P$ is not an equilibrium point and if $\epsilon$ is small enough, $\sigma$ moves outside its first position.

When it is possible to choose $\epsilon$ small enough so that $\sigma$ never overlaps its first position again, we shall call $P$ a wandering point. Let the set of all wandering points be called $W$. If $\sigma$ overlaps itself when $t$ has increased from $t_{0}$ to $t_{0}+\tau$, it has to do so when $t$ has decreased from $t_{0}$ to $t_{0}-\tau$, and conversely.

Thus we arrive at the same set of points $W$ if we let the time decrease instead of increase in the above definition. If $P$ is a wandering point, so are all the points of $\sigma$, since $\sigma$ is open; thus $W$ is open, and the set $M_{1}=M-W$ is closed. For any point of $M_{1}$, any corresponding molecule $\sigma$ will overlap itself after any period of time as $t$ increases (and decreases), no matter how small the molecule may be.

If a given point $P$ is in $M_{1}$, then so is all of the motion through $P$ for decreasing $t$.

For consider any point $(P)_{t}, t<0$, and any molecule $\sigma$ about $(P)_{t}$. Take a molecule $\sigma^{\prime}$ about $P$ so small that $\left(\sigma^{\prime}\right)_{t}$ lies wholly in $\sigma$. Then $\sigma^{\prime}$, hence $\left(\sigma^{\prime}\right)_{t}$ and therefore $\sigma$, intersects itself after any period of time, and thus $(P)_{t}$ is in $M_{1}$. The same reasoning shows that any unique curve of motion lies wholly in $W$ or in $M_{1}$.

Similarly we define $M_{2}$ as the set of non-wandering points with respect to $M_{1}, M_{3}$ as the set of non-wandering points with respect to $M_{2}$ and so forth. Thus we obtain an ordered set of closed sets $M, M_{1}, M_{2}, \cdots, M_{\omega}, M_{\omega+1}, \cdots, M_{r}$. We shall call the limiting set, $M_{r}$, the set of central motions in analogy with the ordinary definition.

(c). By the customary argument it is possible to prove the following property. 
When the time increases and decreases every wandering point approaches $M_{1}$ uniformly.

Furthermore if $P$ is a point of $M_{1}$, it will either stay indefinitely in $M_{1}$, or leave $M_{1}$. In the latter case it becomes a wandering point and must nevertheless approach $M_{1}$ later. By the usual argument we obtain the following result. The probability that an arc of a curve of motion lies inside of an arbitrary neighborhood of $M_{1}$ approaches unity uniformly as the interval of time for such an arc increases indefinitely.

(d). Let us call, as usual, the limiting points of a curve of motion for increasing $t, \omega$ points, and its limiting points for decreasing $t, \alpha$ points.

The $\alpha(\omega)$ limit points $A$ of a motion $C$ are formed of complete motions. It is sufficient to show, for a fixed $t_{0}>0$, that if $P$ is a point of $A$, then there is a point $P^{\prime}$ of $(P)_{t_{0}}$ in $A$; the property is evident for $t_{0}<0$. If $Q$ is a point of $C$, then there are points $Q_{\tau_{1}}, Q_{\tau_{2}}, \cdots$ of $C$ lying in $(Q)_{\tau_{1}},(Q)_{\tau_{2}}, \cdots$ respectively and approaching $P$. The points $Q_{\tau_{1}+t_{0}}, Q_{\tau_{2}+t_{0}}, \cdots$ of $C$ must then approach the point set $(P)_{t_{0}}$. They have a limit point $P^{\prime}$ in the set; thus $P^{\prime}$ is a point of $A$ in $(P)_{t_{0}}$, as required.

The limit points of any set of complete motions consist of complete motions.

The proof is exactly as above except that the points $Q_{\tau_{1}}, Q_{\tau_{2}}$, ... may lie on different motions. Instead of the sequence $M_{1}$, $M_{2}, M_{3}, \cdots$ above, used to define the central motions $M_{r}$, we can use another set as follows. Let us call $N_{1}$ the set formed by the $\alpha$ limit points of all motions on $M$ together with their geometrical limit points. It is easy to see that $M_{1} \supset N_{1}$. Similarly we define $N_{2}$ on $N_{1}$, etc. The limiting set $N_{s}$ consists of those motions which belong to their own $\alpha$ limit points, say $E$, and the geometrical limiting points of $E$.

(e). Evidently $N_{s} \subset M_{r}$; are they identical?* The set of central points contained in a small molecule $\sigma$ must overlap itself in $M_{r}$ for $t_{0}<0$ however large $t_{0}$ and however small $\sigma$ may be. Hence there exists in $\sigma$ a pair of central points $P_{1}$ and $Q_{1}$, positions reached by the same point after a certain period of time. We shall assume that $Q_{1}$ precedes $P_{1}$.

Choose about $P_{1}$ a neighborhood $\sigma_{1}$ so small that both $\sigma_{1}$ and $\left(\sigma_{1}\right)_{t_{1}}$, the corresponding neighborhood of $Q_{1}$, together with

* See G. D. Birkhoff and P. A. Smith, loc. cit., p. 354. 
their limit points shall be contained in $\sigma$. There exist in $\sigma_{1}$ two central points $P_{2}$ and $Q_{2}$ about which we can choose two molecules $\sigma_{2}$ and $\left(\sigma_{2}\right)_{t_{2}}$ completely interior to $\sigma_{1}, Q_{2}$ preceding $P_{2}$. Continue in this manner. We can choose the molecules $\sigma_{k}$ such that

(a) the diameter of $\sigma_{k}$ converges to zero as $k \rightarrow \infty$, and

(b) the periods of time $t_{k}$ increase indefinitely.

Then the limiting point $L$ of the molecules is interior to every molecule $\sigma_{k}$, and thus the curve of motion $C$ through $L$ for decreasing $t$ has the following property $\left(C\right.$ belongs to $M_{r}$ ). For every $k,(L)_{t_{k}}$ has a point in $\sigma_{k-1}$; thus $L$ is an $\alpha$ limit point of $C$ Moreover, as has been shown above, through $L$ exists a complete curve of motion lying in the set of $\alpha$ limit points of $C$ and hence lying in $M_{r}$ and its own set of $\alpha$ limit points. Therefore we may make the following statement.

The complete motions lying in their own set of $\alpha$ limit points are everywhere dense on $M_{r}$. It readily follows that through every central point passes a complete motion lying wholly in $M_{r}$.

(f). It must be noticed that if a motion $C$ has a common point with its $\alpha$ limit points and its $\omega$ limit points it does not necessarily belong to either one. However a motion lying in its set of $\alpha$ limit points and having a common point with its set of $\omega$ limit points is also part of the latter, and is pseudo-recurrent according to the usual definition.

The pseudo-recurrent motions and the recurrent motions, these being defined in the customary way, belong of course to $M_{r}$. Furthermore, in the present case, it is easy to see that $M_{1}$ and $N_{1}$ are not equivalent.

Take on the sphere a simple closed curve* $C$ through an equilibrium point $P$, and let $Q$ be a unique point of Peano on $C$. Let us assume that the motions through $Q$ have as $\omega$ limit points a periodic motion $C_{1}$, for the motions lying on the righthand side, $P$ of course for $C$, and another periodic motion $C_{2}$ for the motions lying on the left-hand side. The point $P$ is the only equilibrium point between $C_{1}$ and $C_{2}$. It is easy to see that $P Q$ belongs to $M_{1}$, but not to $N_{1}$.

3. Central Motions on the Sphere. It is well known that if on

* This example was pointed out by $\mathrm{H}$. Whitney; he also proved independently that $M_{r}$ consists of complete motions. 
the sphere a curve of motion $C$ possesses the two following properties:

(1) $C$ never crosses itself;

(2) on $C$ there is a point $P$ such that $C$ for indefinitely increasing (or decreasing) $t$ has always points arbitrarily near $P$; then through $P$ passes a periodic motion.

It immediately follows from a theorem in $\$ 2$ that such periodic points $P$ are everywhere dense on the central motions, provided that we consider only the part of $M_{r}$ which is not the set of equilibrium points. Thus we may make the following statement.

On a sphere $M$, the central motions $M_{r}$ consist of the equilibrium points, the periodic motions, and their limit points.

These limit points form boundaries of regions; therefore the central motions which are not periodic terminate in equilibrium points or sets of equilibrium points and are, then, nowhere dense on $M_{r}$.

A periodic motion through a point $P$ is the only central motion issuing from $P$ since two periodic motions cannot intersect, and any other central motion, being terminated in equilibrium points for decreasing $t$, has no common points with a periodic motion. Moreover if $P$ is a central, non-periodic point, there exist periodic points converging to $P$ not lying on $H(P)$ (the set of curves of motion through $P$ ); thus there exist at most two different sequences of periodic motions converging to $P$, and thus to at most two different central motions through $P$. Evidently the recurrent and pseudo-recurrent motions are the equilibrium points and the periodic motions.

4. Possibility of Choice. Consider a point $P$ and the set of the $\omega$ limit points of all the curves of motions through $P$, say $\Omega_{\omega}(P)$. If $P_{1}$ is a position reached by $P$ at some later time, we have

$$
\Omega_{\omega}(P) \supset \Omega_{\omega}\left(P_{1}\right) .
$$

The number of possible choices of $P$ cannot increase; in particular, if $P$ and $P_{1}$ are on the same periodic motion,

$$
\Omega_{\omega}(P)=\Omega_{\omega}\left(P_{1}\right) \text {. }
$$

Consider now a point $P$ on a central motion $C$ on a sphere and suppose that, at $P$, the curve of motion is not unique. Let $C_{1}$ be 
another curve of motion through $P$. Consider the arc $P_{1} Q_{1}$ of $(P)_{\epsilon}$, where $P_{1}$ lies on $C$ and $Q_{1}$ on $C_{1}$. Since a motion passing through $P_{1} Q_{1}$ and not passing through $P_{1}$ or $Q_{1}$ cannot be a central motion, we define thus a small region $P P_{1} Q_{1}$ characteristic of the central motion $C$, or possibly of the two central motions $C$ and $C_{1}$. Therefore we have the following result.

The central motions, except possibly the equilibrium points, from which may issue at least one other motion form at most a denumerable set.

If there exist two periodic motions $C_{1}$ and $C_{2}$ (or any two closed curves made up of motions of $M$ ) such that the region $r$ bounded by $C_{1}$ and $C_{2}$ does not contain any equilibrium points in its interior, it is necessary, in order that some point $P$ of $r$ can choose to approach in the future either $C_{1}$ or $C_{2}$, that there be a unique periodic motion $c$ in $r$.

If $P$ is interior to $r, c$ cannot coincide with $C_{1}$ or $C_{2}$. Moreover, $P$, as long as it stays on $c$, will be able to go to any point of $r$ at almost any time, more precisely at a time $T, t-\tau \leqq T \leqq t+\tau$, $t$ being arbitrarily chosen and $\tau$ the period on $c$. The above condition is also sufficient if $c$ contains a point of Peano. To prove the first statement, take a point $P_{0}$ of $r$ having two curves $c_{1}$ and $c_{2}$ asymptotic to $C_{1}$ and $C_{2}$ as $t$ increases. Of course, the motion $c$ through $P_{0}$ for decreasing $t$ cannot be asymptotic to either $C_{1}$ or $C_{2}$. Let us draw two closed bands $B_{1}$ and $B_{2}$ of width $\epsilon$ including $C_{1}$ and $C_{2}$. Let $P_{1}$ and $P_{2}$ be the first points on $c_{1}$ and $c_{2}$ respectively in common with $B_{1}$ and $B_{2}$. By cutting the region $R\left(R=r-\left(B_{1}+B_{2}\right)+\right.$ boundary $)$ by the arc $P_{1} P_{0} P_{2}$, we obtain a simply connected region which does not contain any equilibrium points; therefore $c$ cannot be interior to this region. If we choose $\epsilon$ suitably, $c$ has no common points with $B_{1}$ and $B_{2}$ and thus it has a common point $Q$ with $P_{1} P_{0} P_{2}$. Hence following $c$ further, we run into $P_{0}$ again, and obtain thereby a periodic motion. By the same argument, it is seen that through every point of $r$ passes a motion issuing from $P_{0}$; thus $c$ is the only periodic motion in $r$.

The motion $c$ cuts $r$ into two regions $r_{1}$ and $r_{2}$. Every point on $c$ can pass to any point of $r$, while a point in terior to, say, $r_{1}$, must be in $B_{1}$ after a certain interval of time, and can never reach a point of $r_{2}$. The sufficiency of the condition is not hard to prove.

HARVARD UNIVERSITY 